\title{
Evaluation of Efficacy of Endovenous Radio Frequency Ablation for Varicose Veins in Industrial Workers-A Prospective Study
}

\author{
Venkata Pavan Kumar Karanam ${ }^{1}$ Nandkishor Sopan Rao Sude ${ }^{1} \quad$ Prasanth Gurijala $^{1}$ \\ Ravi Kumar Teppa ${ }^{1}$ Madan H. S. ${ }^{1}$ \\ ${ }^{1}$ Department of General Surgery, ESIC Medical College and Hospital, \\ Hyderabad, Telangana, India \\ Int J Recent Surg Med Sci 2022;8:103-108. \\ Address for correspondence Venkata Pavan Kumar K., MS, \\ Department of General Surgery, ESIC Medical College and Hospital, \\ Sanath Nagar, Hyderabad, Telangana, India \\ (e-mail: pavankmc@gmail.com).
}

\begin{abstract}
\section{Keywords}

- Doppler ultrasound

- radio frequency ablation

- varicose veins

Introduction The disease of varicose veins is a common vascular condition affecting one out of five in world population. Although conventional surgical treatments were considered the gold standard, they were fraught with many complications. Endovenous thermal interventions like endovenous radio frequency ablation (RFA) have largely superseded traditional surgery as the gold standard treatment as it is minimally invasive. Our initial experience with this treatment modality is presented along with the review of literature.

Aim Our aim was to evaluate the efficacy, safety, clinical outcomes, and health economic benefits of endovenous RFA for varicose veins in our setup.

Materials and Methods It was a prospective observational study conducted by the Department of General surgery at ESIC Medical College and Hospital, Hyderabad, India, from September 2020 to February 2021. Thirty consecutive patients with primary varicose veins were evaluated by detailed history, clinical examination, and venous Doppler study of the lower limbs. The procedure was performed under spinal anesthesia and Doppler ultrasound guidance using the ClosureFast (Medtronic) radio frequency segmental ablation system. Additionally, multiple stab phlebectomies were performed to deal with tributary varices in required cases. Various intraoperative (length of vein ablated and operative time) and postoperative (complications and return to work) variables were studied. Postoperative follow-up at 1,8,24 weeks was done clinically and using Doppler ultrasound to look for the recanalization of vein. The collected data were entered in Microsoft Excel spreadsheet and analyzed using descriptive statistics.

Results The mean (standard deviation) age of patients was 39.1 (8.7) years. Ten patients had C2 disease, six C3, eleven C4, and three had C6 disease. The mean postoperative visual analog scale score on day 1 was 1.4 . Mean return to normal activity time was 1.3 days. Average return to work time was 2.1 days. The post-procedure total occlusion rate at 24 weeks follow-up was $96 \%$.

Conclusion Endovenous RFA is a safe and effective minimally invasive modality for treating lower limb varicose veins, providing health economic benefits to patients.
\end{abstract}

DOI https://doi.org/ $10.1055 / \mathrm{s}-0042-1742629$. ISSN $2455-7420$. (c) 2022. Medical and Surgical Update Society. All rights reserved. This is an open access article published by Thieme under the terms of the Creative Commons Attribution-NonDerivative-NonCommercial-License, permitting copying and reproduction so long as the original work is given appropriate credit. Contents may not be used for commercial purposes, or adapted, remixed, transformed or built upon. (https://creativecommons.org/ licenses/by-nc-nd/4.0/)

Thieme Medical and Scientific Publishers Pvt. Ltd., A-12, 2nd Floor, Sector 2, Noida-201301 UP, India 


\section{Introduction}

Varicose veins represent a common health problem, the effects of which in terms of disability and health care costs are considerable. Although the prevalence of varicose veins is higher among Indian population, ${ }^{1}$ generally the patients ignore to seek out treatment at early stages. Hence, the patients undergoing surgical management represent only the tip of iceberg. Modern surgical methods for varicose veins were pioneered by Friedrich Trendelenburg, toward the latter half of the 18th century, and his surgical principles of treatment are still accepted as the "gold standard. ${ }^{2}$ However, in association with technological advances, there has been continual research into treating the disease using minimally invasive methods like endovenous RFA, laser ablation, subfascial endoscopic perforator surgery, etc. Numerous studies have validated the endovenous method as an excellent minimally invasive alternative to conventional surgical methods. ${ }^{3,4}$ However, these modalities are not easily available owing to their high cost and need for expertise, especially in public-funded institutes.

Our institute is a public-funded institute which mainly caters health care needs of industrial workers. Industrial workers whose job requires a long duration of standing and heavy weightlifting are more prone to developing varicose veins. ${ }^{5-8}$ Hence, in our setup, the prevalence of varicose veins in patients attending general surgery clinic was higher than that of the general population. Most of our patients were presenting with complications of varicose veins like lipodermatosclerosis, ulcer, and bleeding which resulted in poor performance at work. ${ }^{9}$ The conventional surgical method in these population results in delay in their return to work owing to its high postoperative pain levels and wound complications. ${ }^{10}$ This, in turn, can significantly delay their returning to work and affect their income. Hence, this study was undertaken as a pilot study to evaluate the efficacy, safety, clinical outcomes, and health economic benefit to the patients with the use of endovenous RFA. The results of this study were expected to guide us in standardizing this procedure in our setup.

\section{Materials and Methods}

A prospective study of 30 consecutive patients presenting to the outpatient department of general surgery for the treatment of primary varicosities was undertaken at ESIC Medical College and Hospital, Sanath Nagar, Hyderabad, India, from the period of September to September 2021. Clinically, patients were classified based on Clinical-Etiology-Anatomy-Pathophysiology (CEAP) classification. Inclusion criteria were age group of 20 to 60 years, clinical scales C2 to C6, primary etiology, and great saphenous vein (GSV) and short saphenous vein anatomical territory, with reflux as the underlying pathology. Patients with superficial or deep vein thrombosis, aneurysms, pacemakers, and recurrent varicose veins were excluded from the study. A preoperative duplex ultrasound (DUS) examination of the venous system for the target lower limb was performed by a radiologist for each patient to identify the condition of deep veins, compe- tency of the saphenofemoral junction (SFJ), characteristics of truncal vein, competency of saphenopopliteal junction (SPJ), and perforator incompetence using established protocols. The severity of SFJ incompetence was also graded as per the established criteria. ${ }^{11}$ This study was conducted in adherence to the declaration of Helsinki.

All the procedures were performed by two general surgeons with experience in performing vascular ultrasounds. Informed consent was obtained from every patient. The procedure was performed under spinal anesthesia with all ideal conditions to prevent vasospasm. Under intraoperative ultrasound guidance, an access site to target GSV was identified, and the vein was percutaneously cannulated with $18 \mathrm{G}$ needle. Through the needle, a 0.018-inch guidewire was inserted in the GSV; thereafter, the needle was removed, and a $7 \mathrm{Fr} \times 11-\mathrm{cm}$ introducer sheath was advanced over the guidewire. Then, the guidewire was removed, and a $7 \mathrm{Fr}$ Closure Fast (Medtronic, United States) RFA catheter with $7 \mathrm{~cm}$ heating element was introduced through the sheath. Under ultrasound guidance, after demonstrating the typical "Mickey Mouse" appearance of the common femoral artery, common femoral vein, and great saphenous vein, the catheter was advanced till the tip and was placed $2 \mathrm{~cm}$ below the SFJ or at the ostium of the superficial epigastric vein, whichever was proximal (-Fig. 1). Tumescent anesthesia was manually delivered in the fascial envelope of GSV, using a 24G spinal needle (-Fig. 2). Thereafter, the vein was segmentally ablated with pre-set energy settings in the radio frequency generator confirmed with auditory feedback (-Fig. 3). Simultaneously, intraoperative ultrasound imaging was used to confirm vein shrinkage and increase in vein wall echogenicity. Multiple phlebectomies were then performed to tackle the tributary varices in the required cases. All the wounds were dressed, and the treated limb was wrapped in an elastic crepe bandage.

Postoperatively, an analgesic was administered, on a required basis. All patients were encouraged to ambulate as soon as they had recovered from the spinal anesthesia and were discharged on postoperative day 1 or 2 , with an instruction to wear below-knee Class II graduated compression stockings for 2 weeks. Postoperatively, at 1,8, and 24 weeks, the patients were examined in the outpatient department, clinically and with DUS, for any immediate and late postoperative complications. The occlusion of the GSV trunk and persistent pain scores were recorded.

\section{Statistical Analysis}

The data were analyzed using IBM SPSS Statistics for Windows, Version 27.0. Armonk, NY United States: IBM Corp, and descriptive statistics were expressed as a number and a percentage for qualitative variables and as mean standard deviation (SD) for quantitative variables.

\section{Results}

A total of 30 patients were enrolled for this study. The group comprised of 25 males and five females. The mean (SD) age of 


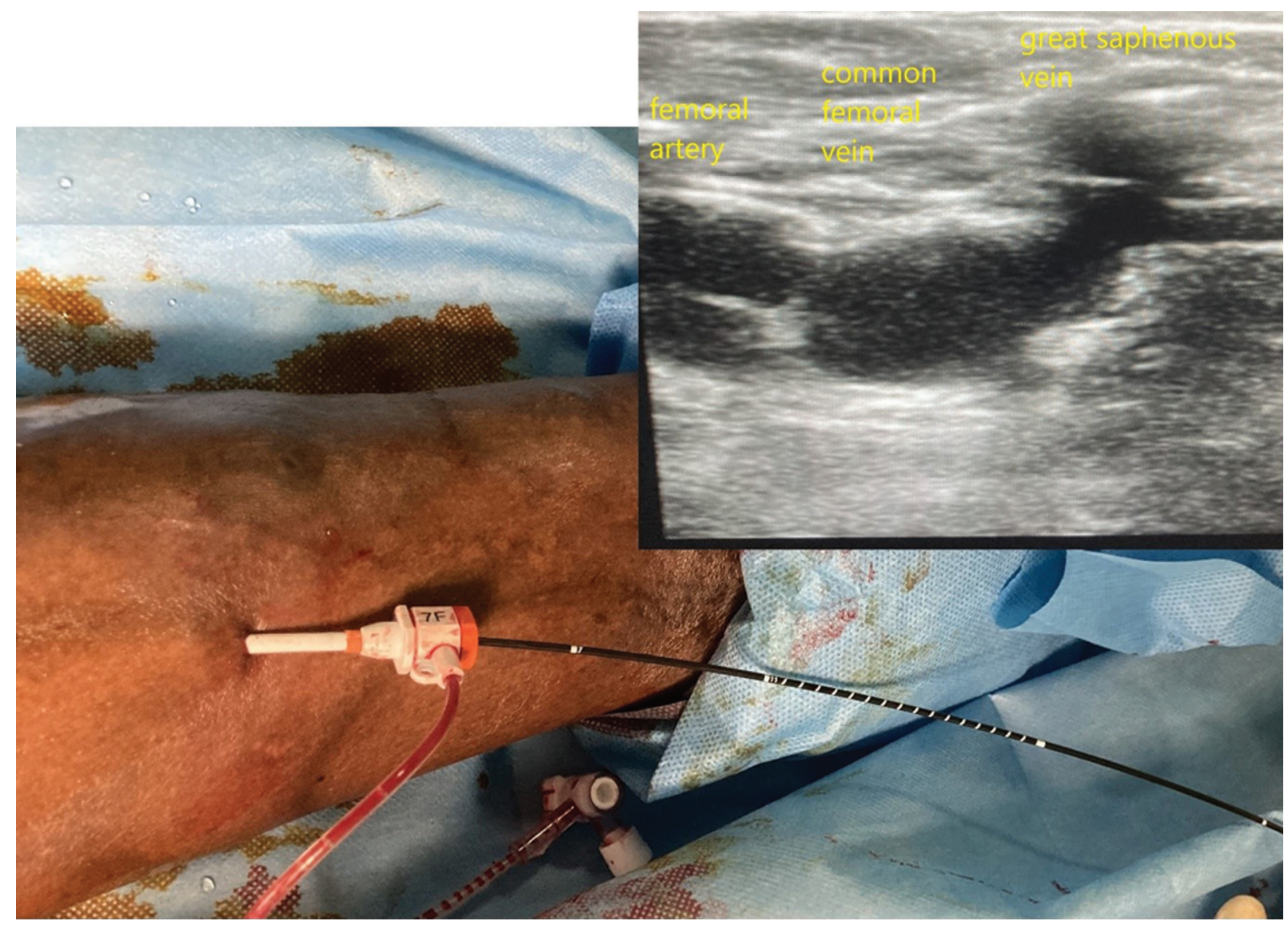

Fig. 1 Introducer sheath with RFA catheter inside. Inset: Duplex ultrasound image demonstrating the typical "Mickey Mouse" appearance of the common femoral artery, common femoral vein, and great saphenous vein.

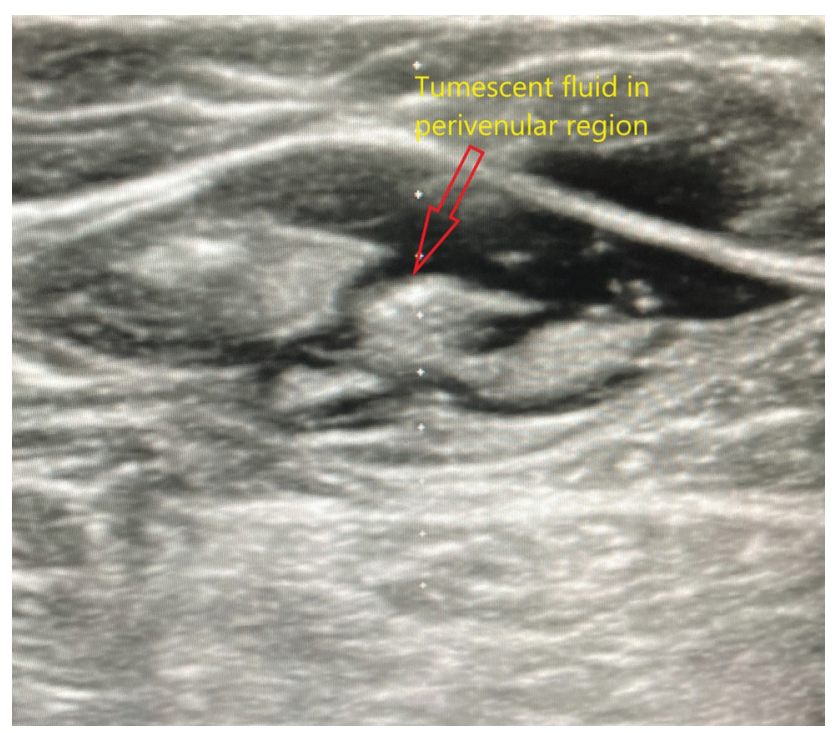

Fig. 2 Duplex ultrasound image demonstrating injection of tumescent fluid around great saphenous vein.

patients was 39.1 (8.7) years. In 18 patients, right limb was treated, and in 12 patients, left limb was treated ( $\mathbf{- T a b l e ~} \mathbf{1}$ ). When evaluated as per the CEAP clinical classification, ten patients were in $\mathrm{C} 2$, six in $\mathrm{C} 3$, eleven in $\mathrm{C} 4$, zero in $\mathrm{C} 5$, and three in C6 (-Fig. 4). On preoperative DUS, SFJ valvular incompetence was seen in all patients, SPJ incompetence in

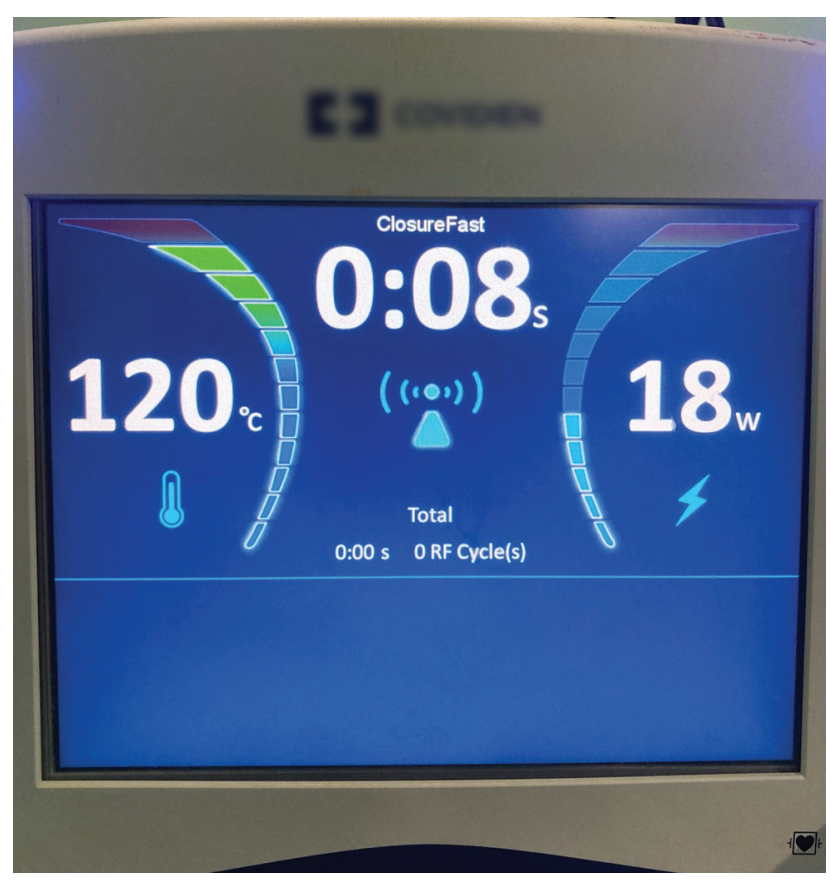

Fig. 3 RFA generator depicting energy settings. RFA, radio frequency ablation. one patient, and concomitant perforator incompetence in 18 patients (-Fig. 5). On DUS, zero patients had grade 1 SFJ reflux,11 patients had grade 2 reflux, 16 patients had grade 3 reflux, and 3 patients had grade 4 reflux (-Fig. 6). The 
Table 1 Demographic variables

\begin{tabular}{|l|l|l|l|}
\hline Variable & & No. of patients & Percentage (\%) \\
\hline \multirow{3}{*}{ Sex } & Male & 25 & 83.3 \\
\cline { 2 - 4 } & Female & 5 & 16.6 \\
\hline \multirow{4}{*}{ Age(y) } & $20-30$ & 6 & 20 \\
\cline { 2 - 4 } & $30-40$ & 14 & 46.6 \\
\cline { 2 - 4 } & $40-50$ & 6 & 20 \\
\cline { 2 - 4 } & $50-60$ & 4 & 13.3 \\
\hline \multirow{3}{*}{ Limb } & Right & 18 & 60 \\
\cline { 2 - 4 } & Left & 12 & 40 \\
\hline
\end{tabular}

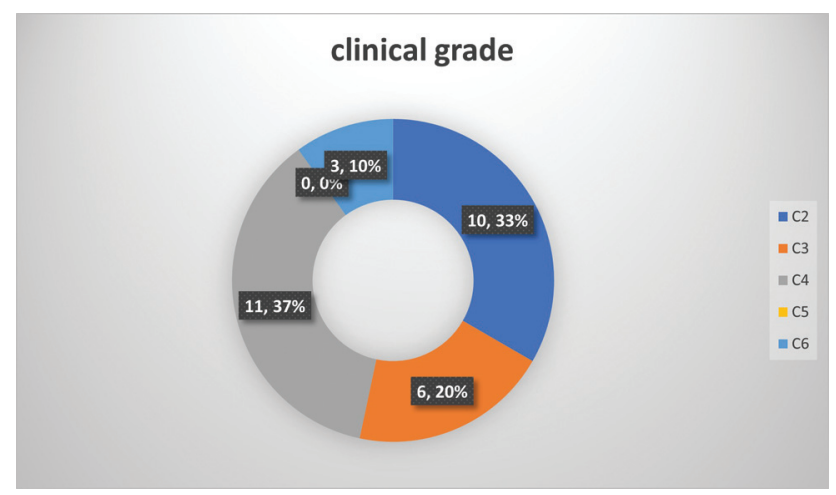

Fig. 4 Distribution of patients as per clinical grade (C) of CEAP classification. CEAP, Clinical-Etiology-Anatomy-Pathophysiology.

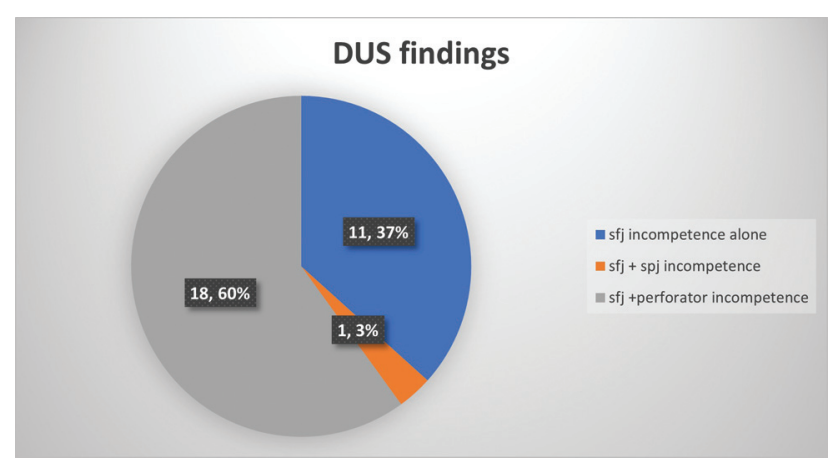

Fig. 5 Distribution of Doppler ultrasound findings across patients.

average length of GSV that was ablated using RFA was $39.8 \mathrm{~cm}$, and the mean operative time was 52 minutes. Post-ablative vein wall thickening was noted in all patients.

The mean postoperative visual analog scale (VAS) score on day 1 was 1.4. Mean to return to normal activity time was 1.3 days. Average return to work time was 2.1 days. Postoperatively thermal skin burns were noted in one patient over a length of $5 \mathrm{~cm}$ skin over ablated vein due to insufficient tumescent fluid. Two patients had bruising over the thigh which required additional analgesics ( - Table 2 ). None of the patients had paraesthesia over limbs, and there was no occurrence of deep venous thrombosis in any patient. On 6 month follow-up, only one patient had recanalization of

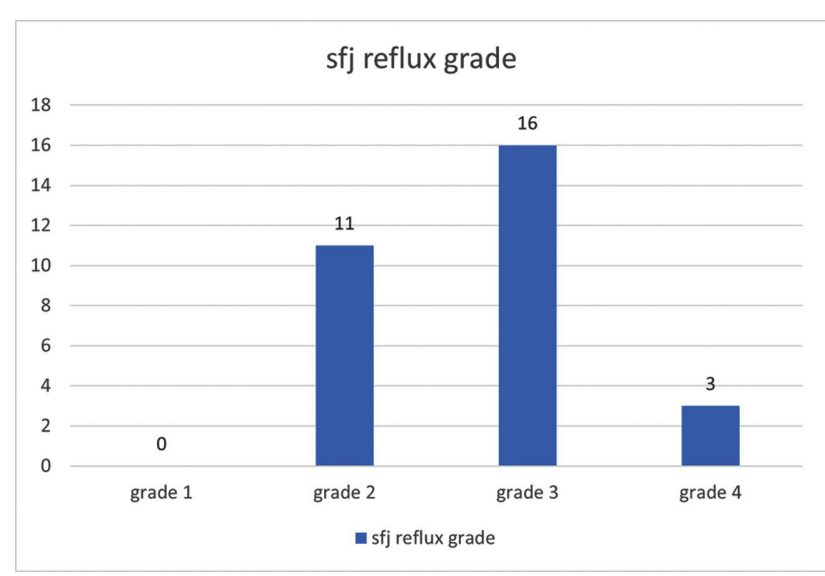

Fig. 6 Distribution of SFJ reflux grades across patients. SFJ, saphenofemoral junction.

Table 2 Postoperative complications

\begin{tabular}{|l|l|l|}
\hline Complication & No. of patients & Percentage (\%) \\
\hline Skin burns & 1 & 3.3 \\
\hline Bruising & 2 & 6.6 \\
\hline Paraesthesia & 0 & 0 \\
\hline DVT & 0 & 0 \\
\hline
\end{tabular}

Abbreviation: DVT, deep venous thrombosis.

GSV. The postprocedural total occlusion rate of target GSV at 6 months follow-up was $96 \%$. The patient with recanalization was $\mathrm{C} 6$ category, and his ulcer showed no signs of improvement. In the other 2 patients of 66 category complete ulcer healing was achieved. On 6 month follow-up, none of the patients complained of significant paraesthesia and limb pain. Except one patient with recanalization, rest all were satisfied with improvement in the quality of their lives.

\section{Discussion}

The disease of varicose veins is one of the common vascular conditions encountered by general surgeons. Varicose veins are part of a wide spectrum of disorders caused by underlying chronic venous hypertension and are associated with significant quality-of-life impairment. Many observational studies have demonstrated that most adults have reticular or thread veins, whereas varicose veins or more severe stages of venous disease (CEAPC2-C6) are present in 25 to $40 \%$ of the population..$^{5,6,12}$ Venous disease is more common in highly industrialized rather than less industrialized countries, possibly due to differences in lifestyle and activity. ${ }^{7,8}$ This observation has indeed formed the basis of our study as our institute mainly caters industrial workers.

Optimal patient management involves a detailed holistic patient assessment, evaluation of patient expectations, and minimally invasive, multimodal therapy to address underlying hemodynamic abnormalities and reduce venous hypertension. Largely driven by an appreciation of the slow recovery and suboptimal outcomes after traditional varicose 
vein operations and patient desire for less invasive interventions, there has been an explosion of endovenous procedures in the past 15 years. Endovenous thermal ablation (EVTA) of varicose veins was introduced in the late 1990s with RFA. ${ }^{13}$ Initial continuous pull back RFA systems showed varied long-term occlusion rates. ${ }^{13}$ The present-day systems employ the segmental ablation method with feedback mechanism (either auditory or visual) for optimal energy delivery to the tissues and result in higher long-term occlusion rates.

Many studies with reliable data of 5 years or longer have validated the long-term durability of these procedures. ${ }^{14}$ For thermal ablation procedures, early outcomes have proved to be an accurate surrogate marker for long-term treatment success. As a result, endovenous thermal interventions have largely superseded traditional surgery as the gold standard treatment for superficial venous reflux., Based on published studies, early outcomes (pain, bruising, and return to normal activity and work) after endovenous procedures are superior to traditional varicose vein surgery. Our study achieved similar results in all the above early outcomes on par with the available literature. In a study conducted by Bozoglan et al, ${ }^{15}$ after RF ablation, mean postoperative VAS score was 1.4 , mean return to normal activity time was 1.3 days, and mean return to work time was 2.1 days. In our study, these values are 1.7, 1.1 days, 2.3 days, respectively.

A study conducted by Almeida et al (RECOVERY STUDY) ${ }^{16}$ showed a statistically lower incidence of minor complications and no major complications in RFA patients. No major adverse postoperative events were noted in our study. Minor complications were observed in $10 \%$ of patients. Skin burns which are unique complications to EVTA modalities were observed in one patient probably due to inadequate tumescent anesthesia.

In a multicenter cohort study conducted by Proebstle et al, $^{14}$ vein occlusion rates at 6 months and 5 years were 98.6 and $91.9 \%$, respectively. In our study, the occlusion rate was $96 \%$ at 6 month follow-up. One patient with venous ulcer had recanalization with persistent ulcer. Of the three patients with venous ulcer treated in our study, two patients had complete ulcer resolution (-Figs. 7 and 8). Reassuring results of our study in terms of safety and vein occlusion rates make RFA an effective modality in treating varicose veins especially in the purview of general surgeons. Our patients achieved significant health economic benefits in view of early return to work due to faster recovery and reduced loss of person days. This promising outcome helped us to standardize the procedure in treating larger proportion of our patient population with higher prevalence of varicose veins compared with the general population.

\section{Limitations}

This study is an observational study with small sample and short follow-up period. A study with a larger sample and longer follow-up is needed to measure the long-term treatment success. Also, comparative studies with other modalities are required to compare the outcomes.

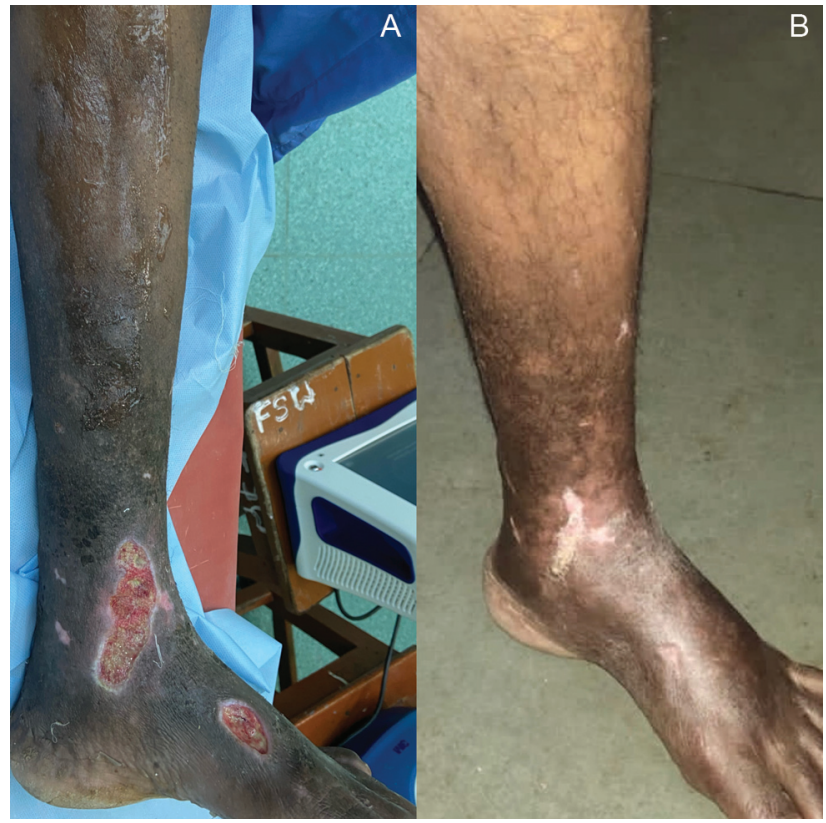

Fig. 7 (A) Pre-ablation photo of our patient with chronic non-healing ulcer and severe lipodermatosclerosis changes. (B) Post-ablation photo after 3 month follow-up shows healed ulcer and significant reduction in lipodermatosclerosis.

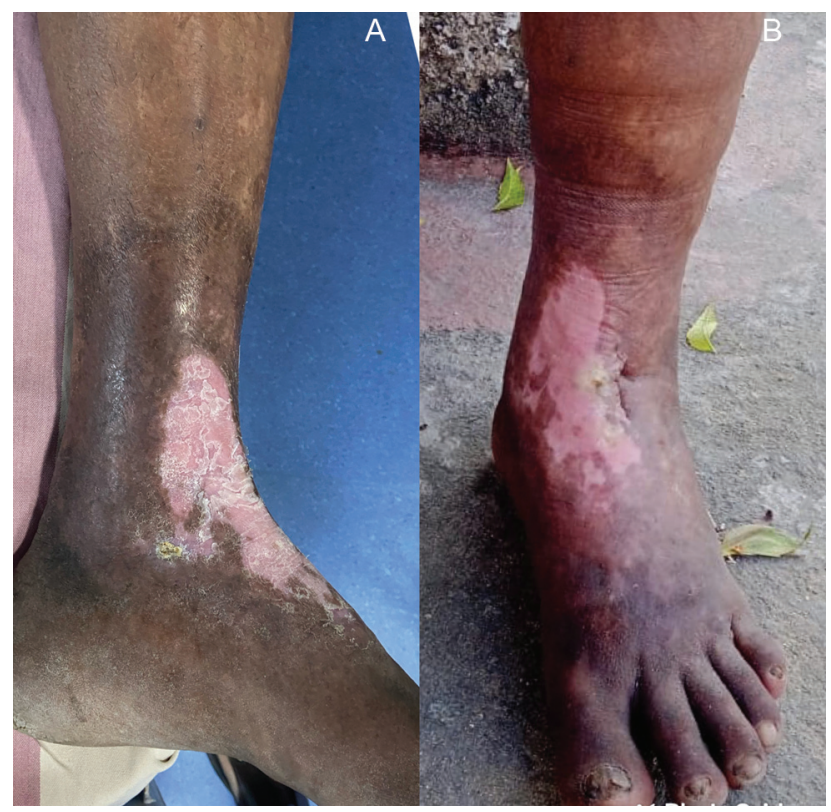

Fig. 8 (A) Post-ablation photo of our patient at 1 month follow-up showing healed ulcer (patient had a chronic non-healing ulcer). (B) A 10 month follow-up showing reduced lipodermatosclerosis and stable healed ulcer.

\section{Conclusion}

Endovenous RFA is a safe and effective minimally invasive modality for treating primary varicose veins. Our results are on par with the available literature. Postoperative early return to work results in considerable health economic benefits to the patients. Training general surgeons in this minimally invasive procedure may benefit larger proportion 
of patients suffering from varicose veins especially in publicfunded institutes. ${ }^{17-23}$

\section{Conflict of Interest}

None declared.

\section{References}

1 Malhotra SL. An epidemiological study of varicose veins in Indian railroad workers from the South and North of India, with special reference to the causation and prevention of varicose veins. Int J Epidemiol 1972;1(02):177-183

2 Raffetto JD, Eberhardt RT. Chronic venous disorders: general considerations. In: Johnston WK, Corenwett JL, eds. Rutherford's Vascular Surgery. 7th ed. Philadelphia: Elsevier; 2010 pp. 831-44

3 Nesbitt C, Bedenis R, Bhattacharya V, Stansby G. Endovenous ablation (radiofrequency and laser) and foam sclerotherapy versus open surgery for great saphenous vein varices. Cochrane Database Syst Rev 2014;(07):CD005624. Doi: 10.1002/14651858

4 Brittenden J, Cotton SC, Elders A, et al. A randomized trial comparing treatments for varicose veins. N Engl J Med 2014; 371(13):1218-1227

5 Rabe E, Guex JJ, Puskas A, Scuderi A, Fernandez Quesada F, Coordinators VCPVCP Coordinators. Epidemiology of chronic venous disorders in geographically diverse populations: results from the Vein Consult Program. Int Angiol 2012;31(02):105-115

6 Maurins U, Hoffmann BH, Lösch C, Jöckel KH, Rabe E, Pannier F. Distribution and prevalence of reflux in the superficial and deep venous system in the general population-results from the Bonn Vein Study, Germany. J Vasc Surg 2008;48(03):680-687

7 Escudero Rodríguez JR, Fernández Quesada F, Bellmunt Montoya S. Prevalence and clinical characteristics of chronic venous disease in patients seen in primary care in Spain: results of the international study Vein Consult Program. Cir Esp 2014;92(08): 539-546

8 Robertson LA, Evans CJ, Lee AJ, Allan PL, Ruckley CV, Fowkes FG. Incidence and risk factors for venous reflux in the general population: Edinburgh Vein Study. Eur J Vasc Endovasc Surg 2014;48(02):208-214

9 Mallick R, Lal BK, Daugherty C. Relationship between patientreported symptoms, limitations in daily activities, and psychological impact in varicose veins. J Vasc Surg Venous Lymphat Disord 2017;5(02):224-237

10 Kanwar A, Hansrani M, Lees T, Stansby G. Trends in varicose vein therapy in England: radical changes in the last decade. Ann R Coll Surg Engl 2010;92(04):341-346
11 Choi J, Hong S, Park S, Park C, Seol H, Cha I. Grading of saphenofemoral junction incompetence in varicose vein: color Doppler ultrasonographic findings. Radiological Society of North America 2005 Scientific Assembly and Annual Meeting, November 27 December 2, 2005, Chicago IL

12 Callam MJ. Epidemiology of varicose veins. Br J Surg 1994;81(02): 167-173

13 Whiteley MS, Shiangoli I, Dos Santos SJ, Dabbs EB, Fernandez-Hart TJ, Holdstock JM. Fifteen year results of radiofrequency ablation, using VNUS closure, for the abolition of truncal venous reflux in patients with varicose veins. Eur J Vasc Endovasc Surg 2017;54 (03):357-362

14 Proebstle TM, Alm BJ, Göckeritz O, et al. Five-year results from the prospective European multicentre cohort study on radiofrequency segmental thermal ablation for incompetent great saphenous veins. Br J Surg 2015;102(03):212-218

15 Bozoglan O, Mese B, Eroglu E, Ekerbiçer HC, Yasim A. Comparison of endovenous laser and radiofrequency ablation in treating varices in the same patient. J Lasers Med Sci 2017;8(01):13-16

16 Almeida JI, Kaufman J, Göckeritz O, et al. Radiofrequency endovenous ClosureFAST versus laser ablation for the treatment of great saphenous reflux: a multicenter, single-blinded, randomized study (RECOVERY study). J Vasc Interv Radiol 2009;20(06):752-759

17 Metta JR, Mehra R, Jaiswal SS, Bhagwat AR, Singh G. Evaluation of radiofrequency ablation for primary varicose veins: a preliminary study. Indian J Vasc Endovasc Surg 2019;6:37-41

18 Badham GE, Strong SM, Whiteley MS. An in vitro study to optimise treatment of varicose veins with radiofrequency-induced thermo therapy. Phlebology 2015;30(01):17-23

19 Ramamurthy V, Channabasappa RG. Clinical presentation and surgical management of varicose veins. Int J Anat Radiol Surg 2021;10(03):SO30-SO33

20 Rasmussen LH, Lawaetz M, Bjoern L, Vennits B, Blemings A, Eklof B. Randomized clinical trial comparing endovenous laser ablation, radiofrequency ablation, foam sclerotherapy and surgical stripping for great saphenous varicose veins. Br J Surg 2011;98 (08):1079-1087

21 Goodyear SJ, Nyamekye IK. Radiofrequency ablation of varicose veins: best practice techniques and evidence. Phlebology 2015;30 (2, Suppl):9-17

22 Belentsov SM, Veselov BA, Chukin SA, Ektova MV, Makarov SE. [Criteria for selection of patients for radiofrequency ablation of major venous trunks in varicose disease, short- and long-term outcomes]. Angiol Sosud Khir 2013;19(04):177-181

23 Lurie F, Creton D, Eklof B, et al. Prospective randomised study of endovenous radiofrequency obliteration (closure) versus ligation and vein stripping (EVOLVeS): two-year follow-up. Eur J Vasc Endovasc Surg 2005;29(01):67-73 